\title{
On the vanishing of the measurable Schur cohomology groups of Weil groups
}

\author{
C. S. Rajan
}

\begin{abstract}
We generalize a theorem of Tate and show that the second cohomology of the Weil group of a global or local field with coefficients in $\mathbb{C}^{*}$ (or, more generally, with coefficients in the complex points of an algebraic torus over $\mathbb{C}$ ) vanish, where the cohomology groups are defined using measurable cochains in the sense of Moore. We recover a theorem of Labesse stating that the admissible homomorphisms of a Weil group to the Langlands dual group of a reductive group can be lifted to an extension of the Langlands dual group by a torus.
\end{abstract}

\section{Introduction}

A classical theorem of Tate asserts the vanishing of the Schur cohomology group (see [Ser]),

$$
H_{\mathrm{gc}}^{2}\left(G_{K}, \mathbb{C}^{*}\right)=\{1\}
$$

where $G_{K}$ is the absolute Galois group of a global or local field $K$ and $G_{K}$ acts trivially on $\mathbb{C}^{*}$. The cohomology groups are those that occur in Galois cohomology, constructed using continuous cochains with respect to the profinite topology on $G_{K}$ and the discrete topology on $\mathbb{C}^{*}$. The obstruction to lifting a projective representation of a finite or a profinite group $G$ to a linear representation of $G$ lies in the Schur cohomology group $H^{2}\left(G, \mathbb{C}^{*}\right)$. Consequently, Tate's theorem asserts that any projective representation $\tilde{\rho}: G_{K} \rightarrow P G L_{n}(\mathbb{C})$ of $G_{K}$ has a lift to a continuous, linear representation of $\rho: G_{K} \rightarrow G L_{n}(\mathbb{C})$, satisfying $\pi \circ \rho=\tilde{\rho}$, where $\pi: G L_{n}(\mathbb{C}) \rightarrow P G L_{n}(\mathbb{C})$ denotes the natural projection.

A natural question that arises is to establish analogues of Tate's theorem and the lifting theorem for projective representations for more general 'Galois type' groups. For example, instead of $G_{K}$, we can consider the Weil group $W_{K}$ associated to a global or local field $K$ (or, more generally, the complex points of the conjectural Langlands group, the continuous representations of which parametrize automorphic representations). These are topological groups which are no longer profinite. Since our primary motivation is the application to lifting, we need to work with a cohomology theory where the second cohomology $H^{2}(G, A)$ parametrizes the topological extensions of $G$ by $A$. Such a cohomology theory has been defined and developed by Moore using measurable cochains [Moo64a, Moo64b, Moo76a, Moo76b]. We show that the analogue of Tate's theorem holds in this framework.

Theorem 1. Let $K$ be a local or global field, and let $W_{K}$ denote the Weil group of $K$. Then the Moore cohomology group $H^{2}\left(W_{K}, \mathbb{C}^{*}\right)$ vanishes, where the Weil group $W_{K}$ acts trivially on $\mathbb{C}^{*}$, and $\mathbb{C}^{*}$ is equipped with the usual Euclidean topology.

More generally, let $T$ be an algebraic torus over $\mathbb{C}$ with a continuous action $G_{K} \rightarrow \operatorname{Aut}(T)$, where $\operatorname{Aut}(T)$ is the group of algebraic automorphisms of $T$. We let $W_{K}$ act on $T$ via the canonical

Received 23 September 2002, accepted in final form 23 January 2003.

2000 Mathematics Subject Classification 11R34 (primary), 22E55 (secondary).

Keywords: Weil groups, measurable cohomology, Schur cohomology groups, lifting projective representations.

This journal is (C) Foundation Compositio Mathematica 2004. 


\section{Cohomology of Weil groups}

morphism to $G_{K}$, and consider $T(\mathbb{C})$ equipped with the usual Euclidean topology as a $W_{K}$ module. Our main theorem is as follows.

THEOREM 2.

$$
H^{2}\left(W_{K}, T(\mathbb{C})\right)=(0)
$$

One of the intriguing aspects of these vanishing theorems, is the comparison with the corresponding Galois cohomology groups. Over number fields it is not true in general that $H_{\mathrm{gc}}^{2}\left(G_{K}, T(\mathbb{C})\right)$ vanishes. Thus the problems arising from the presence of the (real) archimedean places towards the Galois cohomology of complex tori disappear upon going over to the Weil group, and the Weil group of a number field behaves more like the fundamental group of a curve than the Galois group. However, as pointed out by S. Lichtenbaum (see Remark 3), it is not true that the higher cohomology groups $H^{i}\left(W_{K}, T(\mathbb{C})\right)$ vanish for $i \geqslant 3$.

Apart from the intrinsic interest, the principal motivation for proving the vanishing theorem lies in the application to lifting admissible morphisms of the Weil group to the dual Langlands group of a reductive group defined over $K$ (see $\S 8$ ). These applications to lifting have been obtained by Langlands if $K$ is archimedean [Lan89, Lemma 2.10], by Henniart over non-archimedean local field and function fields [Hen80], and by Labesse over number fields [Lab85] (see § 8). For us, the motivation to prove such a lifting result is in the application to characterizing the image of solvable base change [Raj02] for $G L(2)$.

The results of this paper, as pointed out by S. Lichtenbaum and the referee, are of relevance to the study of the values of the Hasse-Weil zeta functions at integers and the concept of Weil-étale topology introduced by Lichtenbaum in this context.

\section{Weil groups}

We briefly recall the basic properties of Weil groups. The construction and study of the basic properties of Weil groups has been carried out in great detail in the notes of Artin and Tate [AT67], and we refer the reader to this book for further details.

Let $K$ denote a local or global field, and let

$$
C_{K}= \begin{cases}J_{K} / K^{*} & \text { if } K \text { is global, } \\ K^{*} & K \text { local. }\end{cases}
$$

The motivation for the Weil group $W_{K}$ is to obtain non-abelian generalizations of the abelian reciprocity law. We require a completion of the diagram

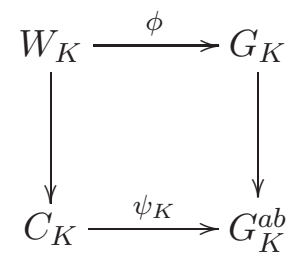

where we require that $W_{K}^{a b} \simeq C_{K}$, and $\psi_{K}: C_{K} \rightarrow G_{K}^{a b}$ is the reciprocity map of class field theory. Here, for a topological group $G, G^{a b}$ denotes the quotient of $G$ by the closure of the commutator group of $G$. A Weil group for $K$ is a triple $\left(W_{K}, \phi,\left\{r_{L}\right\}\right)$ consisting of a topological group $W_{K}$, together with a continuous homomorphism $\phi: W_{K} \rightarrow G_{K}$, with dense image. For each finite extension $L$ of $K$, let $W_{L}=\phi^{-1}\left(G_{L}\right)$. We are given isomorphisms $r_{L}: C_{L} \rightarrow W_{L}^{a b}$, which, composed with the map from $W_{L}^{a b} \rightarrow G_{L}^{a b}$ induced by $\phi$, give the reciprocity map $\psi_{L}$. To be a Weil group, it is further required that these structures be compatible with changing the field, with respect to 


\section{S. RAJAN}

inclusions, norm maps and conjugating by elements of the Galois group, in a manner similar to the behavior of these maps with respect to the reciprocity map in class field theory. In particular, this implies that $W_{L}$ is a Weil group associated to $L$.

Let $K$ now denote a non-archimedean local field or a function field of a curve over a finite field $k$. We refer to these cases as the $\mathbb{Z}$-cases. In the first case, let $N_{K}$ denote the inertia group, and for function fields let $N_{K}$ denote the 'geometric Galois group', i.e. the absolute Galois group $G(\bar{K} / K \bar{k})$ of a separable closure $\bar{K}$ of $K$ over the compositum $K \bar{k}$, where $\bar{k}$ is an algebraic closure of $k$. It is known in these cases that the reciprocity map $\psi_{K}$ is injective, and that the image of $C_{K}$ consists of those elements of $G(\bar{K} / K \bar{k})^{a b}$ which restrict to an integral power of the Frobenius in $G(\bar{k} / k)$. The Weil group admits a similar description in these cases. We have that $W_{K}$ is a dense subgroup of $G_{K}$, and consists of those elements of $G(\bar{K} / K \bar{k})^{a b}$ which restrict to an integral power of the Frobenius in $G(\bar{k} / k)$. There is an exact sequence

$$
1 \rightarrow N_{K} \rightarrow W_{K} \rightarrow \mathbb{Z} \rightarrow 1
$$

The topology on $W_{K}$ comes from the product topology on $N_{K} \times \mathbb{Z}$, where $\mathbb{Z}$ is discrete and $N_{K}$ is a profinite group.

The structure of the Weil group over archimedean fields is known. The Weil group of $\mathbb{C}$ is $\mathbb{C}^{*}$, and the Weil group of $\mathbb{R}$ is an extension of $\mathbb{Z} / 2 \mathbb{Z}$ by $\mathbb{C}^{*}$, where the non-trivial element of $\mathbb{Z} / 2 \mathbb{Z}$ acts by conjugation on $\mathbb{C}^{*}$. The 2-cocycle $a\left(\tau_{1}, \tau_{2}\right)$ defining the extension is given by

$$
a\left(\tau_{1}, \tau_{2}\right)= \begin{cases}1 & \text { if either } \tau_{1} \text { or } \tau_{2} \text { is } 1, \\ -1 & \text { if } \tau_{1}=\tau_{2}=-1 .\end{cases}
$$

For the Weil groups over number fields only a construction is known in terms of the fundamental class, which we now recall. Let

$$
H^{2}\left(G_{K}, C_{\bar{K}}\right)=\underset{\{L: K \subset L \subset \bar{K}\}}{\lim _{\{L}} H^{2}\left(G(L / K), C_{L}\right),
$$

the limit being taken over the finite Galois extensions $L$ of $K$ contained inside a separable algebraic closure $\bar{K}$ of $K$.

One of the main theorems of class field theory is that there exists a 'canonical isomorphism'

$$
\operatorname{inv}_{K}: H^{2}\left(G_{K}, C_{\bar{K}}\right) \rightarrow \mathbb{Q} / \mathbb{Z},
$$

compatible with localization and base change. If $L / K$ is a finite Galois extension of degree $n$, we have an inclusion

$$
H^{2}\left(G(L / K), C_{L}\right) \rightarrow H^{2}\left(G_{K}, C_{\bar{K}}\right)
$$

and the image is the cyclic group $(1 / n) \mathbb{Z} / \mathbb{Z}$ of order $n$. The fundamental class is defined as the element $\alpha_{L / K} \in H^{2}\left(G(L / K), C_{L}\right)$ such that $\operatorname{inv}_{K}\left(\alpha_{L / K}\right)=1 / n$.

Let $W_{L / K}$ be the locally compact topological group defined by the class $\alpha_{L / K} \in H^{2}\left(G(L / K), C_{L}\right)$ :

$$
1 \rightarrow C_{L} \rightarrow W_{L / K} \rightarrow G(L / K) \rightarrow 1 .
$$

Given finite Galois extensions $K \subset L \subset M$, we obtain isomorphisms of Weil groups,

$$
W_{M / L} \simeq \phi^{-1}\left(G_{M / L}\right) \quad \text { and } \quad W_{L / K} \simeq W_{M / K} / W_{M / L}^{\mathrm{c}},
$$

where the notation is that, for any subgroup $H$ of a topological group $G, H^{\text {c }}$ denotes the closure of the commutator subgroup of $H$. It follows from the axioms defining the Weil group that the induced 


\section{Cohomology of Weil groups}

map $C_{M} \rightarrow C_{L}$ is given by the norm map. Further, there is a commutative diagram

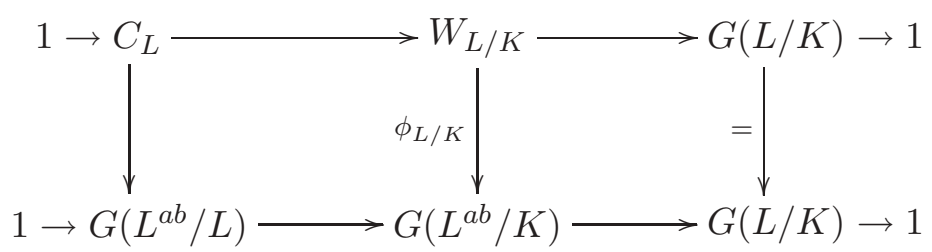

Choosing a transitive system of Weil morphisms, the Weil group $W_{K}$ is obtained as a projective limit,

$$
W_{K}=\lim _{\{L: K \subset L \subset \bar{K}\}} W_{L / K}
$$

The morphism $\phi$ is obtained as the projective limit of the morphisms $\phi_{L / K}$,

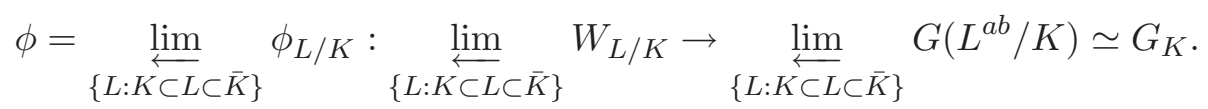

Let $N_{K}$ denote the kernel of $\phi$. Let $D_{L}$ denote the connected component of identity of $C_{L}$. We have an exact sequence given by the reciprocity map,

$$
1 \rightarrow D_{L} \rightarrow C_{L} \rightarrow G\left(L^{a b} / L\right) \rightarrow 1 .
$$

The structure of $D_{L}$ is known. If $L$ has $r_{1}(L)$ real places and $2 r_{2}(L)$ complex places, then

$$
D_{L} \simeq \mathbb{R} \times\left(S^{1}\right)^{r_{2}(L)} \times \mathbb{S}^{r_{1}(L)+r_{2}(L)-1},
$$

where $S^{1}$ denotes the usual circle group, and $\mathbb{S}=(\mathbb{R} \times \hat{\mathbb{Z}}) / \mathbb{Z}$. $\mathbb{S}$ is a 'solenoid', and is a compact, connected, abelian and uniquely divisible group.

Proposition 3. With the above notation:

1) if $K$ is a number field, then $\phi$ is surjective;

2) $N_{K}$ is the connected component of identity in $W_{K}$, and there is an isomorphism

$$
N_{K} \simeq \lim _{\{L: K \subset L \subset \bar{K}\}} D_{L}
$$

\section{Moore cohomology groups}

We recall here the cohomology theory using measurable cochains developed by Moore in a series of papers [Moo64a, Moo64b, Moo76a, Moo76b], especially [Moo76a].

Definition 1. A second countable topological group is said to be polish if its topology admits a separable, complete metric.

Let $G$ be a separable, locally compact group. A $G$-module is a topological abelian group with a continuous action $G \times A \rightarrow A$. Denote by $\mathcal{P}(G)$ the category of commutative polish $G$-modules. For $A \in \mathcal{P}(G)$, let $I(A)=I(G, A)$ be the $G$-module obtained by considering the 'regular representation of $G$ with coefficients in $A$ ': the underlying space of $I(A)$ is the collection of equivalence classes of measurable functions from $G$ to $A$, where $G$ is equipped with a Haar measure. $I(A)$ can be suitably topologized and the resulting topological module is again an element of $\mathcal{P}(G)$.

The existence theorem for a cohomology theory is that there exists a collection of covariant functors $H^{r}(G, \cdot), r \geqslant 0, r \in Z$ from $\mathcal{P}(G)$ to the category of abelian groups satisfying the following. 


\section{S. RAJAN}

C1) For every short exact sequence

$$
0 \rightarrow A^{\prime} \rightarrow A \rightarrow A^{\prime \prime} \rightarrow 0
$$

of modules in $\mathcal{P}(G)$, there is a long exact sequence of cohomology groups,

$$
\cdots \rightarrow H^{r-1}\left(G, A^{\prime \prime}\right) \rightarrow H^{r}\left(G, A^{\prime}\right) \rightarrow H^{r}(G, A) \rightarrow H^{r}\left(G, A^{\prime \prime}\right) \rightarrow \cdots .
$$

C2) $H^{0}(G, A)=A^{G}$, the space of $G$-invariants.

C3) $H^{r}(G, I(A))=0$, for all $r \geqslant 1$ and for all $A \in \mathcal{P}(G)$.

If $H_{1}$ and $H_{2}$ are two such cohomological functors satisfying the above properties $\mathrm{C} 1-\mathrm{C} 3$, it can be shown that there is a unique isomorphism of functors from $H_{1}$ to $H_{2}$ compatible with the given isomorphism in dimension zero.

For the existence part, let $C^{n}(G, A)$ be the set of all Borel functions from $G^{n}$ to $A$. Denote by $\underline{C}^{n}(G, A)$ (also equal to $I\left(G^{n}, A\right)$ ) the set obtained from $C^{n}(G, A)$ by identifying $n$-cochains which agree almost everywhere. Equipped with the usual coboundary operator, the cohomology groups of these two complexes give rise to two cohomological functors $H^{n}(G, A)$ and $\underline{H}^{n}(G, A)$, satisfying $\mathrm{C} 1-\mathrm{C} 3$, and thus are isomorphic. With this isomorphism, the cohomology groups $H^{n}(G, A)$ can be equipped with a topological structure, although not necessarily Hausdorff. If further the topology on $H^{n}(G, A)$ is Hausdorff, then it can be seen that $H^{n}(G, A)$ is a polish group.

Remark 1. Wigner [Wig73] has an alternate approach to these cohomology groups, as Ext functors in a quasi-abelian category in the sense of Yoneda.

The low-dimensional cohomology groups admit a concrete interpretation. Let $Z^{1}(G, A)$ be the collection of continuous 'crossed homomorphisms' $c: G \rightarrow A$ satisfying $c(g h)=h c(g)+c(h)$ for all $g, h \in G$. Denote by $B^{1}(G, A)$ the subset of $Z^{1}(G, A)$ consisting of the 1 -coboundaries of the form $c(g)=g a-a$ for some $a \in A$. Then we have

$$
H^{1}(G, A) \simeq Z^{1}(G, A) / B^{1}(G, A)
$$

In particular, if the $G$-action is trivial on $A$, then $H^{1}(G, A) \simeq \operatorname{Hom}(G, A)$, the group of continuous homomorphisms from $G$ to $A$. In particular, the topology on $H^{1}(G, A)$ is the topology given by uniform convergence on compact subsets, and is thus Hausdorff if $G$ acts trivially on $A$.

For non-abelian coefficients $A$, the space $H^{1}(G, A)$ can be defined in a similar manner (see [Ser80, Annexe, Chapitre VII] and [Moo76a, p. 26]). Let

$$
1 \rightarrow Z \rightarrow M \rightarrow M^{\prime} \rightarrow 1
$$

be an exact sequence of locally compact topological groups, with $Z$ contained in the center of $M$. In exactly the same manner as in the classical case, the following sequence can be shown to be exact in the appropriate sense:

$$
\cdots \rightarrow H^{1}(G, Z) \rightarrow H^{1}(G, M) \rightarrow H^{1}\left(G, M^{\prime}\right) \rightarrow H^{2}(G, Z) .
$$

One of the principal motivations for considering the cohomology theory based on measurable cochains is the isomorphism $H^{2}(G, A) \simeq \operatorname{Ext}(G, A)$, where $\operatorname{Ext}(G, A)$ is the set of equivalence classes of extensions of topological groups

$$
1 \rightarrow A \rightarrow H \rightarrow G \rightarrow 1,
$$

equipped with the Baer product. Here $G$ is a separable locally compact group and $A$ is polish. This follows essentially from a theorem of Dixmier guaranteeing the existence of a Borel measurable cross section $s: G \rightarrow H$ in the situation of Equation (6). In particular, we obtain the following. 


\section{Cohomology of Weil groups}

Proposition 4. Let $G$ be a separable, locally compact group and $A \in \mathcal{P}(G)$. If $H^{2}(G, A)$ is trivial, then any exact sequence of topological groups

splits.

$$
1 \rightarrow A \rightarrow H \rightarrow G \rightarrow 1
$$

There are many comparison theorems of the measurable cohomology theory with continuous cohomology theories. If $G$ is discrete, then the measurable, continuous, and abstract cohomology theories are all isomorphic. It was shown by Wigner [Wig73], that if $G$ is a profinite group and $A$ is a discrete $G$-module, then there is a natural isomorphism for all $i \geqslant 0$,

$$
H_{\mathrm{gc}}^{i}(G, A) \simeq H^{i}(G, A) \text {. }
$$

Consequently, we have the following.

Lemma 1. Let $G$ be a profinite group acting algebraically on a torus $T$ defined over $\mathbb{C}$. Then for $i \geqslant 1$,

$$
H^{i}(G, T(\mathbb{C})) \simeq H_{\mathrm{gc}}^{i}(G, T(\mathbb{C}))
$$

In particular, $H^{2}(G, T(\mathbb{C})) \simeq H_{\mathrm{gc}}^{2}(G, T(\mathbb{C}))$.

Proof. Let $X_{*}(T)$ denote the group of co-characters of $T$. Consider the short exact sequence

$$
1 \rightarrow X_{*}(T) \rightarrow X_{*}(T) \otimes \mathbb{C} \rightarrow T(\mathbb{C}) \rightarrow 1 .
$$

From the long exact sequences associated to the two cohomology theories, and the fact that $H^{1}(G, V)$ vanishes for any vector space $V$ over $\mathbb{C}$ in both the cohomology theories, we obtain, for $i \geqslant 1$,

$$
H_{\mathrm{gc}}^{i}(G, T(\mathbb{C})) \simeq H_{\mathrm{gc}}^{i+1}\left(G, X_{*}(T)\right),
$$

and similarly for the measurable cohomology groups. The lemma then follows by Wigner's comparison theorem.

The cohomology groups constructed by Moore have the added advantage of the presence of a Hochshchild-Serre type spectral sequence. Let $G$ be a locally compact group, $H$ be a closed normal subgroup of $G$, and $A \in \mathcal{P}(G)$.

Proposition 5 [Moo76a, Theorem 9, p. 29]. Assume further that $A$ is locally compact. There is a spectral sequence $E_{r}^{p, q}$ converging to $H^{*}(G, A)$. If further $H^{q}(H, A)$ is Hausdorff, then we have, for any $p$,

$$
E_{2}^{p, q}=H^{p}\left(G / H, H^{q}(H, A)\right) .
$$

In particular, for $q=1$ or 0 , we have $E_{2}^{p, q}=H^{p}\left(G / H, H^{q}(H, A)\right)$.

The presence of the spectral sequence, together with the explicit form of the $E_{2}^{p, q}$ terms for $q=1$ or 0 , implies that the following sequence is exact:

$$
1 \rightarrow H^{1}\left(G / H, A^{H}\right) \stackrel{\inf }{\longrightarrow} H^{1}(G, A) \stackrel{\text { res }}{\longrightarrow} H^{0}\left(G / H, H^{1}(H, A)\right) \rightarrow H^{2}\left(G / H, A^{H}\right) \stackrel{\inf }{\longrightarrow} H^{2}(G, A) .
$$

We require the existence of the spectral sequence in the form of the following corollary.

Corollary 1. In the notation of Proposition 5, assume further the following:

- the differential from $E_{2}^{1,1} \rightarrow E_{2}^{3,0}$ is injective;

- $H^{2}(H, A)=0$;

- the inflation map given by the spectral sequence

$$
H^{2}\left(G / H, H^{0}(H, A)\right) \rightarrow H^{2}(G, A)
$$

is zero.

Then $H^{2}(G, A)=(0)$. 


\section{S. RAJAN}

\section{Proof of Theorem 2 for $\mathbb{Z}$-cases}

We first consider the $\mathbb{Z}$-cases and compare the cohomology of a discrete $G_{K}$-module with the cohomology of the corresponding $W_{K}$-module.

Proposition 6. Let $K$ be a non-archimedean local field or a global field of positive characteristic (Z्Z-cases). Let $A$ be a discrete $G_{K}$-module, and consider $A$ as a $W_{K}$-module via the morphism $\phi$. Then, for $i \geqslant 2$, the restriction map

$$
\phi^{*}: H^{i}\left(G_{K}, A\right) \rightarrow H^{i}\left(W_{K}, A\right)
$$

is an isomorphism.

Proof. We have the following exact sequences of topological groups:

$$
1 \rightarrow N_{K} \rightarrow G_{K} \rightarrow \hat{\mathbb{Z}} \rightarrow 1, \quad 1 \rightarrow N_{K} \rightarrow W_{K} \rightarrow \mathbb{Z} \rightarrow 1
$$

For a profinite group $G$, and a discrete $G$-module, the measurable and continuous cohomology groups are isomorphic [Wig73]. The cohomology group

$$
H^{q}\left(N_{K}, A\right)=\underset{U}{\lim } H^{q}\left(N_{K} / U, A^{U}\right)
$$

is a direct limit of the cohomology groups of the finite groups $N_{K} / U$ as $U$ runs over the family of open subgroups of $N_{K}$. Thus, for $q \geqslant 1$, the cohomology groups $H^{q}\left(N_{K}, A\right)$ are torsion and discrete.

We can apply the spectral sequence, and compute it using the $E_{2}$ term of the spectral sequence. The measurable cohomology groups of $\mathbb{Z}$ are isomorphic to the abstract cohomology groups and thus vanish in degrees greater than one. For $\hat{\mathbb{Z}}$ (see [Ser80, ch. XIII, $\S 1]$ ), the cohomology groups vanish for degrees greater than one. Hence the Hochschild-Serre spectral sequence computing the higher degree cohomology of $G=G_{K}$ or $W_{K}$, with the normal subgroup being $N=N_{K}$, degenerates at the $E_{2}$-stage, and for $n \geqslant 2$ we have the restriction-inflation exact sequences,

$$
1 \rightarrow H^{1}\left(G / N, H^{n-1}(N, A)\right) \rightarrow H^{n}(G, A) \rightarrow H^{0}\left(G / N, H^{n}(N, A)\right) \rightarrow 0 .
$$

Now $H^{1}(\mathbb{Z}, A)=A /(F-1) A$, where $F$ is the image of $1 \in \mathbb{Z}$ in $\operatorname{Aut}(A)$. Using the computation of the cohomology of finite cyclic groups, we obtain, for any discrete $\hat{\mathbb{Z}}$-module $A, H^{1}(\hat{\mathbb{Z}}, A) \simeq$ $A^{\prime} /(F-1) A$, where $A^{\prime}=\left\{a \in A \mid\left(1+F+\cdots+F^{n}\right) a=0\right.$, for some $\left.n\right\}$. Now it can be seen that the torsion part $A_{t} \subset A^{\prime}$ [Ser80]. Hence we have that if $A$ is also torsion as an abelian group, then $H^{1}(\hat{\mathbb{Z}}, A) \simeq H^{1}(\mathbb{Z}, A)$. Since $H^{i}\left(N_{K}, A\right)$ is torsion for $i \geqslant 1$, we obtain the proposition.

Corollary 2. Let $G_{K}$ act algebraically on a torus $T$ defined over $\mathbb{C}$, and let $W_{K}$ act on $T$ via the induced action. Then the cohomology groups $H^{i}\left(W_{K}, T(\mathbb{C})\right)$ vanish for $i \geqslant 2$. In particular, Theorem 2 is true in the $\mathbb{Z}$-cases.

Proof. Consider the exact sequence given by Equation (7). To see that the cohomology groups $H^{i}\left(W_{K}, X_{*}(T) \otimes \mathbb{C}\right)$ vanish for $i \geqslant 2$, we apply the Hochschild-Serre spectral sequence for the Weil group $W_{K}$ and its subgroup $N_{K}$. The only $E_{2}^{p, q}=H^{p}\left(\mathbb{Z}, H^{q}\left(N_{K}, X_{*}(T) \otimes \mathbb{C}\right)\right)$ terms that are possibly non-vanishing are those when $q=0$ and $p=0$ or 1 . Hence, for $i \geqslant 2$, the groups $H^{i}\left(W_{K}, X_{*}(T) \otimes \mathbb{C}\right)$ vanish, and we have $H^{i}\left(W_{K}, T(\mathbb{C})\right) \simeq H^{i+1}\left(W_{K}, X_{*}(T)\right)$. By the above proposition and Lemma 1 , we obtain, for $i \geqslant 2$,

$$
H^{i}\left(W_{K}, T(\mathbb{C})\right) \simeq H^{i}\left(G_{K}, T(\mathbb{C})\right) \simeq H_{\mathrm{gc}}^{i}\left(G_{K}, T(\mathbb{C})\right) .
$$

The corollary then follows from the theorem of Poitou and Tate (see [NSW00]), asserting that the groups $G_{K}$ have strict (Galois) cohomological dimension two. 


\section{Cohomology of Weil groups}

\section{Proof of Theorem 2 for archimedean local fields}

We first prove the following key proposition, which is of use over number fields too.

Proposition 7. Let $H$ be a connected, locally compact abelian group, and $S^{1}$ be the circle group equipped with the trivial action of $H$. Then

$$
H^{2}\left(H, S^{1}\right)=(0) .
$$

Proof. The elements of $H^{2}\left(H, S^{1}\right)$ parametrize equivalence classes of extensions,

$$
1 \rightarrow S^{1} \rightarrow G \rightarrow H \rightarrow 1,
$$

where $S^{1}$ is a closed normal subgroup of $G$, and $H$ is isomorphic to the quotient $G / S^{1}$. We now use a construction due to Hughes [Hug51]. Given a class $\alpha \in H^{2}\left(H, S^{1}\right)$, and an extension corresponding to $\alpha$ as above, choose a measurable cross section $\sigma: H \rightarrow G$, and define for $x, y \in H$

$$
f_{\sigma}(x, y)=\sigma(x) \sigma(y) \sigma(x)^{-1} \sigma(y)^{-1} .
$$

$f_{\sigma}(x, y)$ takes values in $A$, and it can be checked that $f_{\sigma}(x, y)$ is a bilinear, measurable function from $H \times H \rightarrow \mathbb{S}^{1}$. Now by Banach's theorem [Nee97], any measurable homomorphism between two locally compact groups is continuous, and so we obtain a measurable homomorphism from $H \rightarrow \operatorname{Hom}\left(H, S^{1}\right)$, where $\operatorname{Hom}\left(H, S^{1}\right)$ is the space of continuous, unitary homomorphisms of $H$, and hence is topologically isomorphic to the dual group $\hat{H}$ of $H$. But since $H$ is compact abelian, $\hat{H}$ is discrete. By Banach's theorem, the homomorphism $H \rightarrow \hat{H}$ is continuous. Since $H$ is connected, this map has to be the trivial homomorphism.

Hence we have that any extension of $H$ by $S^{1}$ is an abelian, locally compact group. But the identity morphism of $S^{1}$, can be extended to a morphism from $G \rightarrow S^{1}$, and hence any extension splits, and the cohomology group is trivial.

\subsection{Proof for $\boldsymbol{W}_{\mathbb{C}}$}

Corollary 3. Theorem 2 is true for $W_{\mathbb{C}}$.

Proof. This follows from the fact that for any compact group $G$ and a topological vector space $V$, the higher cohomology groups $H^{i}(G, V)$ vanish for $i \geqslant 1$, and the fact that $H^{2}(\mathbb{R}, \mathbb{R})$ vanishes.

\subsection{Proof of Theorem 2 for $W_{\mathbb{R}}$}

We have to show for a $G(\mathbb{C} / \mathbb{R})$-torus $T$, that $H^{2}\left(W_{\mathbb{R}}, T(\mathbb{C})\right)=(0)$. Let $\mathbb{C}_{0}^{*}, S_{0}^{1}$, and $\mathbb{Z}_{0}$ denote respectively $\mathbb{C}^{*}, S^{1}$, and $\mathbb{Z}$ with the trivial action of $\mathbb{Z} / 2 \mathbb{Z}$, and let $\mathbb{C}_{1}^{*}, S_{1}^{1}$, and $\mathbb{Z}_{1}$ denote respectively $\mathbb{C}^{*}, S^{1}$, and $\mathbb{Z}$, where $-1 \in \mathbb{Z} / 2 \mathbb{Z}$ acts by the non-trivial automorphism $z \rightarrow z^{-1}$. From the structure theory of torsion-free indecomposable $\mathbb{Z}[\mathbb{Z} / 2 \mathbb{Z}]$-modules $[\mathrm{CR} 62, \S 74$, ch. XI]), it follows that any torus $T$ with a $\mathbb{Z} / 2 \mathbb{Z}$-action has a $\mathbb{Z} / 2 \mathbb{Z}$-equivariant filtration

$$
T(\mathbb{C})=T_{0}(\mathbb{C}) \supset T_{1}(\mathbb{C}) \supset T_{2} \supset \cdots \supset(1),
$$

such that the subquotients are either the 'invariant' $\mathbb{C}_{0}^{*}$, or the 'anti-invariant' $\mathbb{C}_{1}^{*}$. Thus we can assume that we are in one of these two cases.

We apply the Hochschild-Serre spectral sequence to the exact sequence,

$$
1 \rightarrow \mathbb{C}^{*} \rightarrow W_{\mathbb{R}} \rightarrow \mathbb{Z} / 2 \mathbb{Z} \rightarrow 1 .
$$

By Proposition 7 , we have that $E_{2}^{0,2}=H^{0}\left(\mathbb{Z} / 2 \mathbb{Z}, H^{2}\left(\mathbb{C}^{*}, T(\mathbb{C})\right)=0\right.$. From the existence of the spectral sequence, it follows that we have to show the following for $j=0,1$ :

$(\mathrm{SS} 1)$ The differential $d^{0}: H^{0}\left(\mathbb{Z} / 2 \mathbb{Z}, H^{1}\left(\mathbb{C}^{*}, \mathbb{C}_{j}^{*}\right)\right) \rightarrow H^{2}\left(\mathbb{Z} / 2 \mathbb{Z}, H^{0}\left(\mathbb{C}^{*}, \mathbb{C}_{j}^{*}\right)\right)$ is surjective.

$(\mathrm{SS} 2)$ The differential $d^{1}: H^{1}\left(\mathbb{Z} / 2 \mathbb{Z}, H^{1}\left(\mathbb{C}^{*}, \mathbb{C}_{j}^{*}\right)\right) \rightarrow H^{3}\left(\mathbb{Z} / 2 \mathbb{Z}, H^{0}\left(\mathbb{C}^{*}, \mathbb{C}_{j}^{*}\right)\right)$ is injective. 


\section{S. RAJAN}

The differential $E_{2}^{m-1,1} \rightarrow E_{2}^{m+1,0}$ has been calculated by Hochschild and Serre [HS53, Theorem 4], in the situation when the normal subgroup acts trivially on the coefficients. The negative of the differential is given by taking the cup-product with the class $a \in H^{2}\left(\mathbb{Z} / 2 \mathbb{Z}, \mathbb{C}_{1}^{*}\right)$, given as in Equation $(2)$ and defined by the extension defining $W_{\mathbb{R}}$. Since $\mathbb{C}^{*}$ acts trivially on $T(\mathbb{C})$, we can use this description of the differential, and further $H^{1}\left(\mathbb{C}^{*}, \mathbb{C}_{j}^{*}\right)=\operatorname{Hom}\left(\mathbb{C}^{*}, \mathbb{C}_{j}^{*}\right)$.

We claim the following.

Claim. For any integer $i$, the cup-product

$$
\cup a: \hat{H}^{i}\left(\mathbb{Z} / 2 \mathbb{Z}, \operatorname{Hom}\left(S^{1}, S_{j}^{1}\right)\right) \rightarrow \hat{H}^{i+2}\left(\mathbb{Z} / 2 \mathbb{Z}, S_{j}^{1}\right)
$$

is an isomorphism, where $\hat{H}^{i}$ stands for the Tate cohomology groups.

Write $\mathbb{C}^{*}=S^{1} \times \mathbb{R}$. The cohomology groups $H^{i}(\mathbb{R}, A)$ for any coefficient group $A$ are uniquely divisible, and hence the Tate cohomology groups $\hat{H}^{j}\left(\mathbb{Z} / 2 \mathbb{Z}, H^{i}(\mathbb{R}, A)\right)$ vanish for any integer $j$. Hence the above claim implies the corresponding statement of the claim with the group $S^{1}$ replaced by $\mathbb{C}^{*}$, and the coefficients $S_{j}^{1}$ by $\mathbb{C}_{j}^{*}$ for $j=0,1$. Since for any finite group $G$ and coefficient module $A$, we have a surjection from $H^{0}(G, A) \rightarrow \hat{H}^{0}(G, A)$, it is clear that the claim implies the above desired properties (SS1) and (SS2) of the differential in the spectral sequence, and hence the theorem.

The proof of the claim is an explicit, case-by-case calculation with cup-products. By the definition of the extension defining $W_{\mathbb{R}}$, we have that $-1 \in \mathbb{Z} / 2 \mathbb{Z}$ acts by $z \rightarrow z^{-1}$. Since $\mathbb{Z} / 2 \mathbb{Z}$ acts on $S^{1}$ by $z \mapsto z^{-1}$, we have the following isomorphisms as $\mathbb{Z} / 2 \mathbb{Z}$-modules:

$$
\operatorname{Hom}\left(S^{1}, S_{0}^{1}\right) \simeq \mathbb{Z}_{1} \text { and } \operatorname{Hom}\left(S^{1}, S_{1}^{1}\right) \simeq \mathbb{Z}_{0}
$$

Let $\chi: \mathbb{Z} / 2 \mathbb{Z} \rightarrow \mathbb{Q} / \mathbb{Z}$ be the non-trivial homomorphism, and let $\delta \chi \in H^{2}(\mathbb{Z} / 2 \mathbb{Z}, \mathbb{Z})$ be the image with respect to the connecting homomorphism of the exact sequence $0 \rightarrow \mathbb{Z} \rightarrow \mathbb{Q} \rightarrow \mathbb{Q} / \mathbb{Z} \rightarrow 0$. It follows from Herbrand's theorem that the cup-product

$$
\cup \delta \chi: \hat{H}^{i}(\mathbb{Z} / 2 \mathbb{Z}, A) \rightarrow \hat{H}^{i+2}(\mathbb{Z} / 2 \mathbb{Z}, A)
$$

is an isomorphism for all values of $i . \delta \chi$ is given by the formula

$$
\delta \chi\left(\tau_{1}, \tau_{2}\right)= \begin{cases}1 & \text { if either } \tau_{1} \text { or } \tau_{2} \text { is } 1 \\ -1 & \text { if } \tau_{1}=\tau_{2}=-1\end{cases}
$$

The group $\mathbb{Z} / 2 \mathbb{Z}$ considered as a $\mathbb{Z} / 2 \mathbb{Z}$-module occurs naturally as a quotient of $\mathbb{Z}_{j}$ and as a subgroup of $S_{j}^{1}$ for $j=0,1$. Comparing the formulas in Equation (2) for the cohomology class $a$, thought of as an element in $H^{2}\left(\mathbb{Z} / 2 \mathbb{Z}, S_{1}^{1}\right)$, with those for $\delta \chi$, we obtain a commutative diagram,

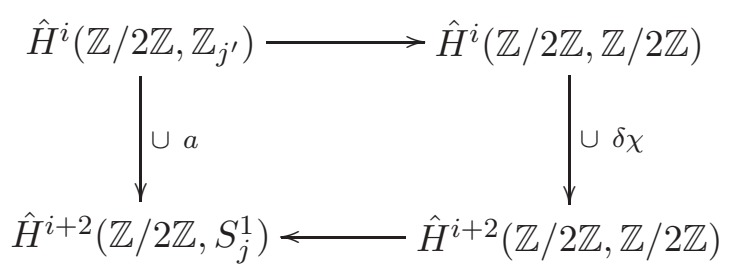

where $j^{\prime} \neq j$ and $j^{\prime}, j \in\{0,1\}$. In order to prove the claim, we have to show that the left-hand vertical map is an isomorphism. From the formulas giving the Tate cohomology groups of cyclic groups as in [CF67, p. 108], we obtain natural isomorphisms

$$
\begin{aligned}
& \hat{H}^{0}\left(\mathbb{Z} / 2 \mathbb{Z}, \mathbb{Z}_{1}\right) \simeq \hat{H}^{0}(\mathbb{Z} / 2 \mathbb{Z}, \mathbb{Z} / 2 \mathbb{Z}) \simeq \hat{H}^{0}\left(\mathbb{Z} / 2 \mathbb{Z}, S_{0}^{1}\right) \\
& \hat{H}^{1}\left(\mathbb{Z} / 2 \mathbb{Z}, \mathbb{Z}_{0}\right) \simeq \hat{H}^{1}(\mathbb{Z} / 2 \mathbb{Z}, \mathbb{Z} / 2 \mathbb{Z}) \simeq \hat{H}^{1}\left(\mathbb{Z} / 2 \mathbb{Z}, S_{1}^{1}\right) .
\end{aligned}
$$




\section{Cohomology of Weil groups}

In the other cases, the cohomology groups $\hat{H}^{i}\left(\mathbb{Z} / 2 \mathbb{Z}, \mathbb{Z}_{j^{\prime}}\right)$ and $\hat{H}^{i+2}\left(\mathbb{Z} / 2 \mathbb{Z}, S_{j}^{1}\right)$ both vanish simultaneously. The claim and Theorem 2 for $W_{\mathbb{R}}$ now follows from the commutativity of the above diagram and the fact that the cohomology of cyclic groups are periodic of period two.

Remark 2. It is clear that the properties $(\mathrm{SS} 1)$ and $(\mathrm{SS} 2)$ hold for any $T(\mathbb{C})$ in place of $\mathbb{C}_{0}^{*}$ and $\mathbb{C}_{j}^{*}$, in view of the structure theorem of torsion-free indecomposable $\mathbb{Z}[\mathbb{Z} / 2 \mathbb{Z}]$-modules.

\section{Proof of Theorem 2 for number fields}

We apply the Hochschild-Serre spectral sequence to the exact sequence,

$$
1 \rightarrow N_{K} \rightarrow W_{K} \rightarrow G_{K} \rightarrow 1 .
$$

By Proposition 7, since the solenoid is a compact connected abelian group, we have for any natural numbers $a, b$

$$
H^{2}\left(\mathbb{S}^{a} \times\left(S^{1}\right)^{b}, T(\mathbb{C})\right)=(0),
$$

for the trivial action on $T(\mathbb{C})$. Now $H^{2}(\mathbb{R}, \mathbb{R})$ is zero, and since the second cohomology commutes with projective limits of compact groups [Moo64a, Theorem 2.3], we conclude that

$$
E_{2}^{0,2}=H^{0}\left(G_{K}, H^{2}\left(N_{K}, T(\mathbb{C})\right)\right)=(0) .
$$

In order to prove that the $E_{2}^{2,0}$ does not contribute to the cohomology of the Weil group, we now recall a lemma proved by Langlands [Lan79, Lemma 4] and by Labesse [Lab85, Proposition 5.5(b)] (and providing for us the analogue of Tate's vanishing theorem).

Lemma 2. Let $L$ be a finite extension of $K$ over which $T$ splits. The inflation map

$$
H^{2}\left(G_{L / K}, T(\mathbb{C})\right) \rightarrow H_{\mathrm{ct}}^{2}\left(W_{L / K}, T(\mathbb{C})\right)
$$

is zero, where the $H_{\mathrm{ct}}^{*}$ denotes the continuous cohomology groups of the arguments.

We have the commutative diagram

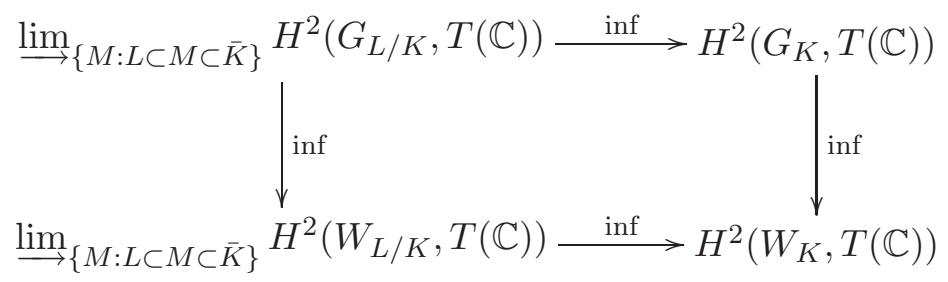

By Proposition 1, the top horizontal map is an isomorphism. Since the left-hand vertical map is zero, we obtain that the inflation map

$$
H^{2}\left(G_{K}, T(\mathbb{C})\right) \rightarrow H^{2}\left(W_{K}, T(\mathbb{C})\right)
$$

is zero.

Finally, we are left with showing that the differential $d_{1}: E_{2}^{1,1} \rightarrow E_{2}^{3,0}$ is injective. We have

$$
\begin{aligned}
& E_{2}^{1,1}=H^{1}\left(G_{K}, \operatorname{Hom}\left(N_{K}, T(\mathbb{C})\right)\right)
\end{aligned}
$$

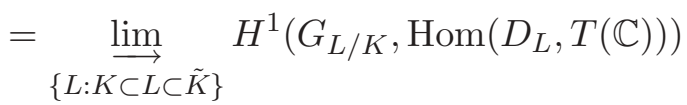

From the structure theorem for $D_{L}$, given by Equation (4), we have

$$
\operatorname{Hom}\left(D_{L}, T\left(\mathbb{C}^{*}\right)\right) \simeq \operatorname{Hom}\left(\mathbb{R} \times \mathbb{S}^{r_{1}(L)+r_{2}(L)-1}, T(\mathbb{C})\right) \oplus \operatorname{Hom}\left(\left(S^{1}\right)^{r_{2}(L)}, T(\mathbb{C})\right) .
$$




\section{S. RAJAN}

Since the first group on the right-hand side of the above equation is uniquely divisible and $G_{L / K}$ is a finite group, we have

$$
H^{1}\left(G_{L / K}, \operatorname{Hom}\left(\mathbb{R} \times \mathbb{S}^{r_{1}(L)+r_{2}(L)-1}, T(\mathbb{C})\right)\right)=(0) .
$$

We need to analyze the structure of $\operatorname{Hom}\left(\left(S^{1}\right)^{r_{2}(L)}, T\left(\mathbb{C}^{*}\right)\right)$ as a $G_{K^{-}}$-module. By passing to a larger extension we can assume that $L$ has no real embeddings. Let $v$ be an archimedean place of $K$. For each archimedean prime $v$ of $K$, fix a prime $w$ of $L$ dividing $v$. Let $G=G_{L / K}$ and let $G_{v}$ be the decomposition group of $G$ at $w$. Denote by $S_{\infty}(K)$ the set of archimedean places of $K$. It can be seen that

$$
\operatorname{Hom}\left(\left(S^{1}\right)^{r_{2}(L)}, T(\mathbb{C})\right) \simeq \oplus_{v \in S_{\infty}(K)} \operatorname{Ind}_{G_{v}}^{G} \operatorname{Hom}\left(S^{1}, T(\mathbb{C})\right) .
$$

Hence, by Shapiro's lemma,

$$
H^{1}\left(G_{L / K}, \operatorname{Hom}\left(\left(S^{1}\right)^{r_{2}(L)}, T\left(\mathbb{C}^{*}\right)\right)\right) \simeq \oplus_{v \in S_{\infty, r}(K)} H^{1}\left(G_{v}, \operatorname{Hom}\left(S^{1}, T(\mathbb{C})\right)\right),
$$

where $S_{\infty, r}(K)$ denotes the set of real places of $K$. In each of the summands we have that complex conjugation $\sigma_{v} \in G_{v}$ acts non-trivially on $S^{1}$. We thus obtain the following commutative diagram:

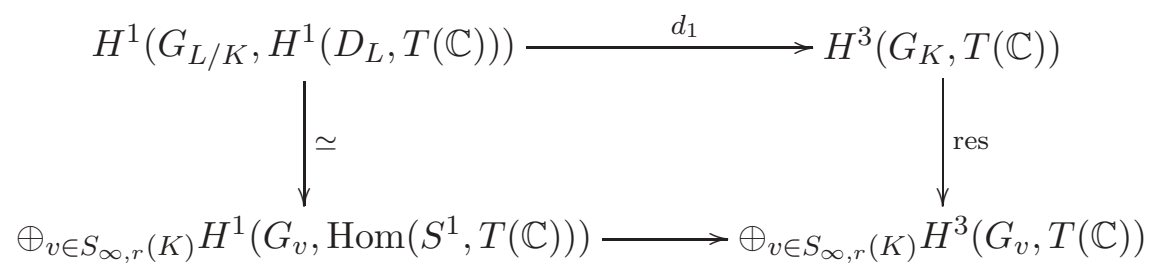

The non-trivial element of the group $G_{v}$, for a real place $v$ of $K$, acts on $T(\mathbb{C})$ by $\sigma_{v}$, and acts on $S^{1}$ by the inverse automorphism. To show that the differential $d_{1}$ is injective, it is enough to show that the bottom arrow is injective. But, for each real place $v$, this map is the corresponding differential (see property (SS2) and also Remark 2) that came up in the computation of $H^{2}\left(W_{\mathbb{R}}, T(\mathbb{C})\right.$ ). Hence, by the computations done in the case of reals, we obtain that this map is injective, and it follows that $E_{3}^{11}=(0)$. This proves Theorem 2 in the case of number fields.

Remark 3. Over number fields, it is not true in general that $H_{\mathrm{gc}}^{2}\left(G_{K}, T(\mathbb{C})\right)$ vanishes. For example, let $K / \mathbb{Q}$ be an imaginary quadratic extension of the rationals and assume that complex conjugation acts by the non-trivial automorphism of $\mathbb{C}^{*}$. It follows from the results of Tate and Poitou [NSW00] that in this situation $H^{2}\left(G_{\mathbb{Q}}, \mathbb{C}^{*}\right) \neq 0$.

It is tempting in the context of the results of this paper to conjecture that the higher cohomology groups $H^{i}\left(W_{K}, T(\mathbb{C})\right)$ vanish for $i \geqslant 3$. However, this conjecture is false, as pointed out by S. Lichtenbaum, even in the simplest situation when $K=\mathbb{C}$. The Weil group is essentially the circle group $S^{1}$ as far as cohomology is concerned when $K=\mathbb{C}$. By Theorem 4 of Wigner [Wig73], the groups $H^{*}\left(S^{1}, \mathbb{Z}\right)$ are isomorphic to the sheaf cohomology groups $H^{*}\left(B S^{1}, \mathbb{Z}\right)$ of the classifying space $B S^{1}$ of $S^{1}$ with values in the constant sheaf with structure group $\mathbb{Z}$ on $B S^{1}$. It is known that the classifying space $B S^{1}$ of the circle group $S^{1}$ is isomorphic to the infinite-dimensional complex projective space $\mathbb{C P}^{\infty}=\varliminf_{n \in \mathbb{N}} \mathbb{C P}^{n}$, and hence we obtain that $H^{i}\left(S^{1}, \mathbb{Z}\right)$ is isomorphic to $\mathbb{Z}$ when $i$ is even and zero otherwise. Thus $H^{i}\left(W_{\mathbb{C}}, \mathbb{C}^{*}\right)$ is isomorphic to $\mathbb{Z}$ when $i$ is odd and vanishes otherwise.

\section{Cohomology of Weil-Deligne groups}

Let $K$ be a local or global field, and let

$$
W_{K}^{\prime}=W_{K} \times S U_{2}
$$




\section{Cohomology of Weil groups}

be the Weil-Deligne group, where $S U_{2}$ denotes the special unitary group in two variables. We consider an action of $W_{K}^{\prime}$ on a torus $T$ defined over $\mathbb{C}$ as before, via the natural projection to $G_{K}$ and letting $G_{K}$ act through a finite quotient as automorphisms of $T$. Consider $T(\mathbb{C})$ to be equipped with the usual Euclidean topology as a $W_{K}$ module. We have the following theorem.

THEOREM 8.

$$
H^{2}\left(W_{K}^{\prime}, T(\mathbb{C})\right)=(0)
$$

Proof. By the results of Moore (see [Moo68] and [Moo76b]), it is known that a topologically simply connected Lie group $G$ satisfies

$$
H^{1}\left(G, S^{1}\right)=(0) \quad \text { and } \quad H^{2}\left(G, S^{1}\right)=(0) .
$$

Since $S U_{2}$ is compact, the cohomology groups $H^{i}\left(S U_{2}, V\right)$ vanish for $i \geqslant 1$ and for any real vector space $V$. Hence we obtain

$$
H^{1}\left(S U_{2}, T(\mathbb{C})\right)=(0) \text { and } H^{2}\left(S U_{2}, T(\mathbb{C})\right)=(0) .
$$

Applying the Hochschild-Serre-Moore spectral sequence, with the normal subgroup as $S U_{2}$, the spectral sequence collapses. Theorem 2 then yields the above theorem.

\section{Application to lifting: Labesse's theorem and base change}

The principal motivation for Theorem 2 is the application to lifting admissible homomorphisms of Weil groups. Let $G$ be a reductive group defined over $K$. Denote by ${ }^{L} G^{0}$ the complex points of the Langlands dual group defined over the complex numbers. The Galois group $G_{K}$ acts on ${ }^{L} G^{0}$, and let ${ }^{L} G$ be the semi-direct product of ${ }^{L} G^{0}$ by $W_{K}$, where $W_{K}$ acts via the natural projection to $G_{K}$. An admissible homomorphism $\phi: W_{K}^{\prime} \rightarrow{ }^{L} G$ consists of the following (for further details refer to [Bor97] and [BR94]):

- $\phi$ is continuous, and composition with the projection to $W_{K}$ induces the identity on $W_{K}$;

- for every $w \in W_{K}, \phi(w)$ is semisimple.

Let $A\left(W_{K}^{\prime},{ }^{L} G^{0}\right)$ denote the collection of admissible homomorphisms. It can be seen that $A\left(W_{K}^{\prime},{ }^{L} G^{0}\right) \subset H^{1}\left(W_{K}^{\prime},{ }^{L} G^{0}\right)$. Assume now that there is an exact sequence of the form

$$
1 \rightarrow S \rightarrow{ }^{L} \tilde{G}^{0} \stackrel{\pi}{\rightarrow}{ }^{L} G^{0} \rightarrow 1
$$

where $G, \tilde{G}$ are as above, $S$ is a central torus in ${ }^{L} \tilde{G}^{0}$, and the morphism $\pi$ is compatible with the action of $G_{K}$. As a corollary of Theorem 2 and the exact sequence Equation (5), we have the following.

Theorem (Labesse [Lab85, Theorem 7.1]). With the notation as above, the natural map

$$
A\left(W_{K}^{\prime},{ }^{L} \tilde{G}^{0}\right) \rightarrow A\left(W_{K}^{\prime},{ }^{L} G^{0}\right)
$$

is surjective.

Remark 4. It is also possible to see this directly from Theorem 2 as follows. Choose a measurable cross section $s:{ }^{L} G^{0} \rightarrow{ }^{L} \tilde{G}^{0}$. Corresponding to an admissible map $\phi \in A\left(W_{K}^{\prime},{ }^{L} G^{0}\right)$, we obtain the pullback of the above extension given by Equation (8):

$$
1 \rightarrow S \rightarrow E \rightarrow W_{K}^{\prime} \rightarrow 1
$$

Hence our main theorem implies the existence of an admissible lift. 


\section{S. RAJAN}

Remark 5. Let $\mathcal{A}$ be a von Neumann algebra realizable on a separable Hilbert space. It follows from Theorem 1 and [Moo76b, Theorem 5, p. 40], that any strongly continuous representation $\rho$ of $W_{K}^{\prime}$ by inner automorphisms of $\mathcal{A}$ is implementable, i.e. there exists a continuous unitary representation $\pi: W_{K}^{\prime} \rightarrow U(\mathcal{A})$, where $U(\mathcal{A})$ is the unitary group in $\mathcal{A}$ such that, for any $a \in \mathcal{A}$ and $w \in W_{K}^{\prime}$, we have $\rho(w)(a)=\pi(w) a \pi(w)^{-1}$. It would be interesting to know of any arithmetical consequences of this result.

\subsection{Characterizing the image of base change}

Following earlier work by Saito and Shintani, Langlands in [Lan80], established the existence of the base change lift $B C_{L / K}$ sending cuspidal automorphic representations of $G L_{2}\left(\mathbf{A}_{k}\right)$ to automorphic representations of $G L_{2}\left(\mathbf{A}_{L}\right)$, provided $L / K$ is a cyclic extension of prime degree. Langlands further characterized the image and the fibres of the base change map $B C_{L / K}$.

The work of Langlands was extended to $G L_{n}, n \geqslant 3$, by Arthur and Clozel [AC89]. The descent property of automorphic representations established by Langlands and Arthur and Clozel is that if $\Pi$ is a cuspidal automorphic representation of $G L_{n}\left(\mathbf{A}_{L}\right)$, which is invariant with respect to the action of the Galois group $G(L / K)$ of a cyclic extension $L / K$ of prime degree, then $\Pi$ lies in the image of the base change map $B C_{L / K}$ from automorphic representations on $G L_{n}\left(\mathbf{A}_{k}\right)$ to automorphic representations on $G L_{n}\left(\mathbf{A}_{L}\right)$. The descent property for invariant representations with respect to a cyclic group of automorphisms was obtained Lapid and Rogawski [LR98] for GL(2), and conditionally by them for $G L(n), n \geqslant 3$.

We assume the functoriality principle, and consider the conjectural complex points of the Langlands group, the representations of which parametrize automorphic representations, as a generalization of the Galois (or Weil) group. We have by Tate's theorem for the Galois group $G_{K}$, and by the main theorem of this paper for the Weil-Deligne group, that $H^{2}\left(W_{K}^{\prime}, \mathbb{C}^{*}\right)=1$. On the functorial side, base change from $K$ to $L$ amounts to restriction to the subgroup $W_{L}$ of an admissible parameter in $A\left(W_{K}^{\prime}, G L_{n}(\mathbb{C})\right)$, since $\left({ }^{L} G L_{n}\right)^{0} \simeq G L_{n}(\mathbb{C})$. Conversely if $\rho$ is a $G(L / K)$-invariant representation of the Galois group $W_{L}$ into $G L_{n}(\mathbb{C})$, then the question of characterizing the image of base change for $G L_{n}$ from $K$ to $L$ translates to the question whether $\rho$ can be extended to a representation of $G_{K}$. If the commutant of the image of $\rho$ consists only of scalars, i.e. if $\rho$ is assumed to be irreducible, then it can be extended as a projective representation of $W_{K}^{\prime}$ to $P G L(n, \mathbb{C})$. By Theorem 1, this projective representation has a lift to $G L(n, \mathbb{C})$. Restricting back to $W_{L}$, it can be checked that the lift differs from the original representation by an invariant character.

In the automorphic context, let $L / K$ be a solvable extension of global fields, and let $\Pi$ be a unitary, cuspidal automorphic representation of $G L_{n}\left(\mathbf{A}_{L}\right)$ which is $\operatorname{Gal}(L / K)$-invariant. It is shown in [Raj02], using the results of Lapid and Rogawski [LR98] (based upon the assumption that a relevant fundamental lemma holds for $n \geqslant 3$, and known to be true when $n=2$ ), that there exists a $G(L / K)$-invariant Hecke character $\psi$ of $K$, and a cuspidal automorphic representation $\pi$ of $G L_{n}\left(\mathbf{A}_{L}\right)$ such that

$$
B C_{L / K}(\pi) \simeq \Pi \otimes \psi
$$

Further, $\psi$ is unique up to base change to $L$ of a Hecke character of $k$.

A difficult case to handle in the proof of this theorem, say for $n=2$, is when $\Pi$ is an automorphically induced representation. In this situation, the automorphic representations are parametrized by admissible parameters of the Weil group, and the lifting theorem helps in producing the required descent (or extension) of the admissible parameter. It can then be checked that the extended parameter gives rise to a cuspidal representation. Although the results of [Raj02] do not use this reasoning to obtain the result in the automorphic context, it is this argument that led us to prove the results of this paper. 


\section{Cohomology of Weil groups}

\section{ACKNOWLEDGEMENTS}

After obtaining a proof of Theorem 1, D. Prasad brought to our attention the results proved by Labesse [Lab85], and I sincerely thank him for this and for useful discussions. I am also indebted to S. Lichtenbaum and the referee for pointing out the applications of the results of this paper to Lichtenbaum's work, and to S. Lichtenbaum for showing me that my earlier conjecture on the vanishing of the higher cohomology groups in question is false.

\section{REFERENCES}

AC89 J. Arthur and L. Clozel, Simple algebras, base change and the advanced theory of the trace formula, Ann. of Math. Studies, vol. 120 (Princeton University Press, Princeton, NJ, 1989).

AT67 E. Artin and J. Tate, Class field theory (Benjamin, New York, 1967).

Bor97 A. Borel, Automorphic L-functions, Proc. Symp. Pure Math., vol. 33, part 2 (American Mathematical Society, Providence, RI, 1977), 27-61.

BR94 D. Blasius and J. D. Rogawski, Zeta functions of Shimura varieties, Proc. Symp. Pure Math., vol. 55, part 2 (American Mathematical Society, Providence, RI, 1994), 525-571.

CF67 J. W. S. Cassels and A. Fröhlich (eds), Algebraic number theory (Academic Press, London; Thompson Book Co., Inc., Washington, 1967).

CR62 C. W. Curtis and I. Reiner, Representation theory of finite groups and associative algebras, Pure and Applied Maths, vol. XI (John Wiley, New York, 1962).

Hen80 G. Henniart, Représentations du groupe de Weil d'un corps local, Enseign. Math. (2) 26(1-2) (1980), 155-172.

HS53 G. P. Hochschild and J.-P. Serre, Cohomology of group extensions, Trans. Amer. Math. Soc. 74 (1953), 110-134.

Hug51 N. J. S. Hughes, The use of bilinear mappings in the classification of groups of class 2, Proc. Amer. Math. Soc. 2 (1951), 742-747.

Lab85 J.-P. Labesse, Cohomologie, L-groupes et fonctorialité, Compositio Math. 55(2) (1985), 163-184.

Lan80 R. P. Langlands, Base change for GL(2), Ann. of Math. Studies, vol. 96 (Princeton University Press, Princeton, NJ, 1980).

Lan79 R. P. Langlands, Stable conjugacy: definitions and lemmas, Can. J. Math. 31(4) (1979), 700-725.

Lan89 R. P. Langlands, On the classification of irreducible representations of real algebraic groups, in Representation theory and harmonic analysis on semisimple Lie groups, Math. Surveys Monogr., vol. 31 (American Mathematical Society, Providence, RI, 1989), 101-170.

LR98 E. Lapid and J. Rogawski, On twists of cuspidal representations of GL(2), Forum Math. 10(2) (1998), 175-197.

Moo64a C. C. Moore, Extensions and low dimensional cohomology of locally compact groups I, Trans. Amer. Math. Soc. 113 (1964), 40-63.

Moo64b C. C. Moore, Extensions and low dimensional cohomology of locally compact groups II, Trans. Amer. Math. Soc. 113 (1964), 64-86.

Moo68 C. C. Moore, Group extensions of p-adic and adelic linear groups, Publ. Math. IHES 35 (1968), $157-222$.

Moo76a C. C. Moore, Group extensions and cohomology for locally compact groups III, Trans. Amer. Math. Soc. 221 (1976), 1-33.

Moo76b C. C. Moore, Group extensions and cohomology for locally compact groups IV, Trans. Amer. Math. Soc. 221 (1976), 35-58.

Nee97 K. H. Neeb, On a theorem of S. Banach, J. Lie Theory 7(2) (1997), 293-300.

NSW00 J. Neukirch, A. Schmidt and K. Wingberg, Cohomology of number fields, Grundlehren der Mathematischen Wissenschaften, vol. 323 (Springer, Berlin, 2000). 


\section{Cohomology of Weil groups}

Raj02 C. S. Rajan, On the image and fibres of solvable base change, Math. Res. Letters 9 (2002), 499-508.

Ser80 J.-P. Serre, Corps locaux, 3rd edn (Hermann, Paris, 1980).

Ser J.-P. Serre, Modular forms of weight one and Galois representations, Collected Papers, vol. III, $292-368$.

Tat77 J. Tate, Number theoretic background, Proc. Symp. Pure Math., vol. 33, part 2 (American Mathematical Society, Providence, RI, 1977), 3-26.

Wig73 D. Wigner, Algebraic cohomology of topological groups, Trans. Amer. Math. Soc. 178 (1973), 83-93.

C. S. Rajan rajan@math.tifr.res.in

Tata Institute of Fundamental Research, Homi Bhabha Road, Bombay 400 005, India 